\title{
Eine epochale "Verwandlung" und mitten darin: Hans Magnus Enzensbergers Geniestreich
}

Der direkt „physikalische“ Blumberg ein älterer Junggeselle wird geschrieben im frühen Jahr 1915. Mithin zeitgleich mit der endgültigen (und geradezu erleichterten) Aufgabe aller Hochzeitspläne, mit der Wiederaufnahme der Wehrdienstabsichten (Kafka will an die Front, sein Mentor, der Chef der Anstalt, lässt das nicht $\mathrm{zu}$, weil er einen seinen vertrautesten Mitarbeiter nicht verlieren will). All dies in jenem Jahr, in dem Kafka den einen „Blutsbruder“ der vergangenen Zeit, den neben Kierkegaard und Kleist: Nämlich Grillparzer, verabschiedet. Denn nun soll es an das Soldat-Werden gehen. Da taugt der Bürokrat nicht mehr zur Identifikation, wie gut er auch immer seine Habsburg verklärenden Dramen geschrieben haben mochte. Jetzt werden übrigens die so zahlreichen Soldatenträume Kafkas geträumt, deren Skala - wahnwitzig! - bis zur Audienz beim kriegserfolgreichen deutschen Kaiser Wilhelm reicht (und die die KafkaForschung mehr oder minder ignoriert hat). Gewiss: Wäre Kafka ein „normaler“ Österreicher gewesen, er hätte verzweifelt sein können über „Kakaniens“ militärische Misserfolge. Das stimmt schon - und eben doch nicht, bei näherer Betrachtung. Es war dieser Autor zutiefst altösterreichisch vielmehr darin, dass er den deutsch-preußischen Alliierten verherrlichte, vor allem dessen Siege an der Ostfront. So kompliziert war das „kakanische“ Seelenleben angelegt, und nicht nur das des Franz Kafka. Sondern auch das der Schnitzler, Hofmannsthal, Musil und Broch, die diesen Krieg ebenfalls erlebten und angesichts der Weltkatastrophe leider nicht immer ganz eindeutig pazifistisch fühlten. Theodor W. Adorno sowie die gesamte „Frankfurter Schule“ wussten es noch: Nur die „Dummen“ erblicken in der Theoriebildung ausschließlich das (ihr andererseits auch wieder notwendige!) spekulative Element. Die Conclusio jedenfalls lautet: In gewisser Weise kann, nein, muss man sagen, dass der Roman als Gattung sich

B. Neumann, Umrisse einer Dritten Kultur im interdisziplinären Zusammenspiel zwischen Literatur und Naturwissenschaft, ELECTRISCHER PROMETHEUS. Umrisse einer Dritten Kultur im interdisziplinären Zusammenspiel zwischen Literatur und Naturwissenschaft, https://doi.org/10.1007/978-3-662-63204-8_21 
mit Kafkas Werk endlich in das neue Zeitalter einpasste, also in ein Weltbild, das von Relativitätstheorie und von der aufkommenden Quantentheorie bestimmt wurde.

Dort aber, dies hat Deutschlands führender Essayist Hans Magnus Enzensberger gefolgert, was an dieser Stelle weiter ausgeführt gehört, ${ }^{1}$ wurde die Physik selbst ,poetisch“. Enzensberger hat nämlich einen hoch informierten und espritvollen Artikel zur (wie immer ungewollten) Nähe der neuesten Quantenphysik zur „Ersten Kultur“ verfasst, der sich zwar auf die Seite der Literatur schlägt, aber in seiner naturwissenschaftlichen Beschlagenheit einen eleganten Brückenschlag darstellt zwischen den beiden Ufern, die noch C. P. Snow so streng getrennt gesehen hatte. In einer Art von Gegenbewegung geschah dies, die ihrerseits einen Paradigmawechsel anzeigte. Will sagen: Die allerneueste Naturwissenschaft ist selbst undenkbar ohne deren (für sie sogar spezifischen!) Anleihen an einer ihrem Ursprung nach ,poetischen“ Metaphorik. Bestimmte Bereiche moderner Poesie wurden und werden geschrieben im entschiedenen Bewusstsein, dass die Welt nach Einstein und nach der auf ihn folgenden Quantentheorie nicht mehr so gesehen werden kann, wie noch zuvor im positivistischen und „mechanistischen“ 19. Jahrhundert. Wenn aber die moderne Mathematik ihrerseits einer „Poesie von Ideen“ zu gleichen beginnt, wie der Kulturwissenschaftler Armand Borel es erkannt zu haben meint (und deshalb als Mottogeber bei Enzensberger beansprucht zu werden vermag), dann muss man bemüht sein, aus Snows „Zwei Kulturen“, aus Lepenies” „Drei Kulturen“, und sowieso aus Feyerabends „unendlich vielen“ Kulturen heraus-, und zurück zu finden zur relativen Einfachheit einer neu zu begründenden „Dritten Kultur“. Was freilich auch meint, dass diese nur in vielfacher Facettierung, und deshalb als interdisziplinäre zu haben sein wird. Mit Enzensberger ginge es dann darum, zu einer neuen, zeitgemässen Osmose zwischen Literatur und Naturwissenschaften zu finden, die der neuen post-newtonischen, weil ,nicht-mechanistischen“ Weltsicht eben dadurch gerecht würde, dass sie sich ihr primär durch die besondere Erkenntnisqualität der Metapher annähern würde.

Hier kommen wiederum Hans Blumenberg und seine „Metaphorologie“ ins Spiel, wie bereits entwickelt; würde ein Friedenspanier hochgestreckt, unter dessen avancierter Vermittlungskapazität dann eine neue, so not- wie aufwendige, weil ihrerseits ganz zeitgemässe Vermittlungsarbeit hin zu einer „Dritten Kultur“

\footnotetext{
${ }^{1}$ Hans Magnus Enzensberger, Die Poesie der Wissenschaft, in: Nomaden im Regal. Essays, Frankfurt/Main 2002, S. 76 ff.
} 
gelingen könnte? Solche Transition scheint nunmehr tatsächlich möglich angesichts neuerer Forschungsarbeiten, wie sie in den vergangenen Jahren unter anderem an Norwegens Technischer Universität in Trondheim geleistet wurden. Die spezifische Potentialität der Metapher wurde darin auch empirisch untersucht, in laborähnlichen Tests an ausgewählten, repräsentativen Lesern. Eine zusammenfassende Studie dazu erschien im Jahr 2019, und seitdem vermag über die Metapher annähernd naturwissenschaftlich exakt gesprochen zu werden. ${ }^{2}$ Das alles besass seine Vorgeschichte: Bereits im Jahr 2011 hatten die Psychologen Paul H. Thibodeau und Lera Boroditski an der renommierten Stanford Universität eine Experimentserie mit insgesamt 485 Studenten unternommen. Denen wurde ein Text vorgelegt, der die Kriminalitätsprobleme in der fiktiven Stadt „Addison“ mithilfe einer (ebenfalls fiktiven) Statistik beschrieb. Die identischen Texte verwendeten lediglich verschiedene Metaphern; einmal war die Kriminalität als „Raubtier“, dann als „Virus“ bezeichnet. Das Ergebnis war verblüffend: Die „Raubtier“-Gruppe schlug intensivierte Verbrecherjagd, Gefängnis und strengere Strafen vor, während die „Virus"-Gruppe die Ursachen der Kriminalität mit Armut-Bekämpfung und besserer Ausbildung bekämpfen wollte. Das Bemerkenswerte daran war, dass ausschliesslich der Gebrauch verschiedener Metaphern, und das auch noch unbewusst für die Probanden, zu ihren so gänzlich verschiedenen Strategien der Kriminalitätsbekämpfung führte. Die geradezu beängstigende Macht der Metaphern schien damit empirisch bewiesen, ihre Zentralstellung bei Aristoteles gerechtfertigt, Blumenbergs Lebenswerk hatte eine empirische Basis gewonnen. Ferner: Unter Rückbezug auf eine in Deutschland 2016 erschienene Untersuchung (Elisabeth Wehling, Politisches Framing. Wie eine Nation sich ihr Denken einredet - und daraus Politik macht) konkludieren die Autorinnen, dass die Metapher eine Art von „superlim i hjernen“, also eine besondere Bindungsfunktion im menschlichen Vorstellungsvermögen darstelle; wobei das Abstrakte konkret gemacht würde, eben im Sinn des griechischen Begriffs (,Übertragung“) und als ein unverzichtbarer und nun sogar experimentell nachweisbarer Vorgang und als erstes Resultat der besonderen sprachlichkognitiven Leistung aller Metaphorik als spezifischer Sprach- und Denkfigur von Alters her. Das geschah an Probanden, denen unter Laborbedingungen vor allem Metaphern aus der politischen Gegenwart (etwa „Flüchtlingsschwemme“ oder „Gutheitstyrannei“) vorgelegt wurden. Die erhebliche Macht der Metapher trat

\footnotetext{
${ }^{2}$ Norunn Askeland/Magdalena Agdestein, Metaforer: Hva, hvor og hvorfor?, Oslo 2019, (Universitetsforlaget).
} 
derart empirisch messbar zutage, vermochte an bestimmten Beispielen bis in die vorbewusste Konditionierung durch beispielsweise die ursprüngliche, prägende Mutterwärme hinein entschlüsselt zu werden (etwa der Ursprung von Metaphern, in denen Wärme mit mütterlicher Liebe, und Bedeutendes mit väterlicher Grösse konnotiert auftraten). ${ }^{3}$ Solche Studien zur empirischen Metaphernforschung markieren ein zentrales desideratum für die Etablierung einer „Dritten Kultur“; sie liefern zudem einen statistisch-empirischen Unterbau zu Blumenbergs lebenslanger Beschäftigung mit der Metapher im kulturellen Prozess als einem Widerspiel zwischen Erkenntnis und Mythenbildung.

Was wiederum bedeutete, dass zwischen dem ,idiot savant“ und dem ,idiot lettré", wie sie sich Enzensberger zufolge seit 200 Jahren unversöhnt gegenüberstehen, erstmals der notwendige Ausgleich zustande käme. Dann erst würden die folgenden Sätze nicht mehr gelten: „Jeder von uns ist bekanntlich fast überall auf der Erde ein Ausländer, und ebenso ist jeder fast auf allen Wissensgebieten ein halber oder ganzer Analphabet. Dies einzusehen ist eine Sache, eine andere ist es, auf den Status des Ignoranten stolz zu sein. Der Shakespeare-Forscher, der nie eine Seite Darwin gelesen hat, der Maler, dem schon schwarz vor Augen wird, wenn von komplexen Zahlen die Rede ist, der Psychoanalytiker, der nichts von den Resultaten der Insektenforscher weiss, und der Dichter, der keinem Neurologen zuhören kann, ohne einzuschlafen - das sind doch unfreiwillig komische Figuren, nicht weit entfernt von einer Art selbstverschuldeter Verblödung!“4 In der Tat. Dieser Tatbestand wird umso bedauerlicher, bedenkt man, dass er seinerseits seit immerhin 200 Jahren existiert. Doch noch zu Lebzeiten von Kafkas „Blutsbruder“ Kleist sah das ganz anders aus, wie bereits entfaltet. Weder die griechische, noch die römische Antike, oder auch die europäische Geschichte danach bis hinein ins 19. Jahrhundert kannten eine solch epochale Aufspaltung der Wissensgebiete; geschweige denn die hier zur Liquidierung anstehende dogmatische Zweiteilung zwischen den Snow'schen „Zwei Kulturen“. Die vieltausendjährigen Kulturgeschichten Chinas (und auch die Indiens) haben sie übrigens in dieser Weise niemals erfahren. Sind darin dem Mythos näher geblieben, als das spätere Europa unter dem Einfluss einer am Ende mechanistischen, so ganz ,positivistisch“ ausgerichteten Aufklärung (wie viele nicht nur technische, sondern auch medizinisch herausragende Entdeckungen diese auch für sich zu verbuchen vermochte, etwa in der Seuchenbekämpfung).

\footnotetext{
${ }^{3}$ Norunn Askeland/Magdalena Agdestein, a. a. O., S. 30 f.

${ }^{4}$ Hans Magnus Enzensberger, Die Poesie der Wissenschaft, a. a. O., S. 77.
} 
Allein der Mythos vermochte die beiden sich immer mehr trennenden Sphären zusammen zu halten, gerade weil er sich zu stützen vermochte auf die ,absolute Metapher“ im Sinne Blumenbergs. „Die Metapher reklamiert eine Ursprünglichkeit, in der nicht nur die privaten und müssigen Provinzen unserer Erfahrung, die Spaziergänger- oder Dichterwelten verwurzelt sind, sondern auch die fachsprachlich verfremdeten Präparatsaspekte theoretischer Einstellung. " ${ }^{5}$ Das ist gewisslich wahr; hier gilt sie noch, die phänomenale (und ihrerseits erzeuropäische) Synthese: „Die vorsokratischen Philosophen sind es gewesen, die in Europa die Physik begründet haben. Empedokles ist der Autor einer dichterischen Kosmologie; und Pythagoras von Samos, dem die Mathematik die legendären Sätze ver-

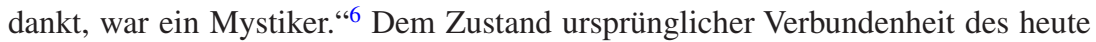
Getrennten aber entsprach, als autochthone literarische Form, das griechischrömisch hergebrachte Lehrgedicht, wie es dann nicht zufällig in der Renaissance wiederbelebt wurde. Nachdem dasselbe die fromme Bigotterie des Mittelalters überstanden hatte, haben in der Renaissance die Maler, Architekten, Philosophen und Naturwissenschaftler, die Cardano, Dürer und Leonardo, Bruno und de Bergerac diesem gehuldigt, indem sie keine Grenzziehung mehr zwischen Poesie und Wissenschaft duldeten. Das galt dann bis weit ins 18. Jahrhundert hinein. Noch Diderot holte sich für seine monumentale Enzyklopädie den Mathematiker d'Alembert an seine Seite. Lichtenberg sowie Goethe dann waren leidenschaftlich naturwissenschaftlich Interessierte, und danach war gerade den Romantikern jede Trennung zwischen dem dichterischen und dem naturwissenschaftlich Bereich nur noch obsolet. Der Tatbestand wurde bereits entfaltet am Beispiel des Friedrich von Hardenberg, der sich Novalis nannte und den Goethe'schen Bildungsroman widerlegen wollte. Ein neuer Mythos sollte begründet werden, aber eben auch einer, der zuvor durch die Aufklärung hindurchgegangen war, jedenfalls im Fall der Jenaer Frühromantiker. „Es spricht alles dafür, dass das grosse Schisma zwischen den Naturwissenschaften auf der einen, den Künsten und den Humaniora auf der anderen Seite eine typische Erfindung des neunzehnten Jahrhunderts ist ...“ Im „Sieg des Positivismus“ liegen „Ursachen und Symptome dieser Entwicklung zugleich ... Der idiot savant und der idiot lettré sind einander ähnlicher, als sie ahnen."

\footnotetext{
${ }^{5}$ Hans Blumenberg, Ausblick auf eine Theorie der Unbegrifflichkeit, zitiert bei Enzensberger, a. a. O., S. 90.

${ }^{6}$ Hans M. Enzensberger, a. a. O., S. 78.

${ }^{7}$ Hans M. Enzensberger, a. a. O., S. 80.
} 
Festzuhalten ist mithin, dass es immer schon, bis hin zur Herrschaft des Positivismus, und nun auch wieder nach seiner Abdankung, ein verbreitetes Bestreben nach Allseitigkeit gab. Erst der Sieg des Positivismus im neunzehnten Jahrhundert brachte die beiden einander ergänzenden „Idioten“ Hans Magnus Enzensbergers hervor. Jeder Erfolg der gegenwärtigen Bestrebungen, eine „Dritte Kultur“ zu etablieren, würde deren Existenz zu einer historischen werden lassen. Franz Kafka wiederum glich spätestens seit seiner Einstein-Begegnung nicht mehr den Schriftstellern des 19. Jahrhunderts, jedenfalls nicht denen des „Poetischen Realismus“ in Deutschland, weder dem erfolgreichen Gustav Freytag, noch dem so bedeutenden Theodor Fontane. Seit der „Blumfeld“Episode samt elektromagnetischem Exempel-Denken stand er viel eher in die Nachfolge Flauberts - und wusste das auch selbst, wenn er eben diesen Sachverhalt mit Gustave Flauberts epistomologischem Roman belegte! Welch eine Selbstreflektion der eigenen revolutionären Schreibposition äusserte sich darin; welch eine Prager Sensibilität für die Eine, die alles umfassende Formel für's neueste Weltverstehen. Die verblüffende Übereinstimmung zwischen Enzensberger und Kafka in Sachen Flaubert führte darüber hinaus direkt zu ihrer objektiv gegebenen Komplizenschaft gegen das, was dann Enzensberger so treffend das „Kasperletheater der Dekonstruktion“ nennen kann, mit Verweis auf die sog. ,Sokal-Affäre“ in den USA. Letztere hatte in dem Nachweis bestanden, dass der postmoderne „Dekonstruktivismus“ jeden Unsinn als Erkenntnis publizierte, sofern der nur verklausuliert und unverständlich genug erschien. Er wurde in den USA noch im vergangenen Jahrhundert geführt von Vertretern der Naturwissenschaften, die einen Unsinns-Artikel einreichten - und als wertvolle Erkenntnis „dekonstruktiven“ Denkens publiziert bekamen, samt glänzenden Bewertungen. Enzensberger soll für uns den Tatbestand zusammenfassen: „Wie ein fernes Echo ihres heissen Bemühens mutet die berüchtigte Sokal-Affäre aus den neunziger Jahren des vergangenen Jahrhunderts an, eine Blamage vergleichbaren Formats, die dem Kasperltheater der Dekonstruktion manchen Buhruf ein-

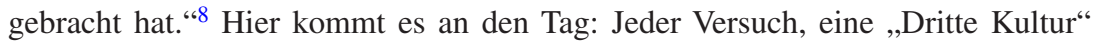
als Neugründung zu etablieren, muss seinerseits die "dekonstruktivistischen“ Beliebigkeiten der vergangenen Postmoderne strikt ausschliessen; muss sie sogar noch, post mortem sozusagen, bekämpfen. Muss sie jedenfalls in ihrer ganzen Haltlosigkeit offenlegen, hat zur Aufgabe, diese selbst zu „dekonstruieren“.

\footnotetext{
${ }^{8}$ Hans M. Enzensberger, a. a. O., S. 80.
} 
Dieser Dämon nämlich lebt noch, gerade wenn er sich totstellt und geschlagen bekennt.

Dieses Gebot der Stunde in mente, kann man zu den bei Enzensberger ebenfalls gesammelten Neubegründungen im Verhältnis zwischen Humaniora und Naturwissenschaften fortschreiten. Ganz neue Grenzüberschreitungen bahnen sich hierin an, alle vergleichbar mit Kafkas ,narrativer Relativitätstheorie" (Axel Gellhaus). Raymond Queneau hat bereits in den sechziger Jahren des vergangenen Jahrhunderts eine wissenschaftliche Enzyklopädie herausgegeben, die Pléiade; und zudem mit Bords ein Buch über die neuere Mathematik geschrieben. Primo Levi und der Pole Stanislav Lem mit seiner Summa technologica von 1963 sind hier ebenso einschlägig, wie später dann Thomas Pynchon mit Gravity's Rainbow von 1973 - einem Buch, das deutsch unter dem Titel Die Enden der Parabel erschien. Mit dem Namen Lem ist auch die konstitutive Nähe dieses Bereichs zur modernen Science Fiction signalisiert. Im Bereich der skandinavischen Sprachen sind Inger Christensen und Lars Gustafsson zu erwähnen, und im Bereich des Deutschen vor allem Durs Grünbein, in dessen Lyrik Physiologie und Neurowissenschaften ihrerseits eine konstitutive Rolle spielen. Man kann konkludieren: Während die Poesie noch immer ein minoritäres Medium darstellt, sind die Naturwissenschaften zu einer (ihrerseits nun auch selbst kulturellen) Übermacht avanciert. Und dennoch, das meint und begründet jedenfalls Enzensberger so überraschend wie überzeugend, verdankt sich dieser Vorgang seinerseits einer spezifischen Osmose der „harten“ mit den ,weichen“"Fächern.

Einige Beispielfälle seien abschliessend aufgerufen, diese Diagnose zu belegen. Samuel Taylor Coleridge, ein Dichter der englischen Romantik, pflegte regelmässig Chemie-Vorlesungen in der Royal Institution zu besuchen. Gefragt, warum er das tue, soll der Dichter geantwortet haben: „Um meinen Vorrat an Metaphern anzureichern.“ Ein interdisziplinäres Vorgehehen, und ein in beide Richtungen exekutierbares! Es hatte sich zudem in nennenswertem Umfang ereignet, blieb freilich weitgehend unbemerkt im aufgewühlten, teilweise auch blutig verfärbten Fahrwasser der P.C. Snow'schen Diskussion um die „Zwei Kulturen“. Enzensbergers Essay enthält doch tatsächlich seitenlange Aufzählungen von verblüffenden Beispielen, auf die hier nur verwiesen werden kann, - während der eine, ganz zentrale, dabei nahezu verstörende Belegfall darin zu finden ist, dass der, heute allseits bekannte, Begriff "Quarks“ von Murray Gell-Mann nach James Joyces' Finnegans Wake benannt, entwickelt (und auch dank des Bekanntheitsgrades der Joyce'schen Literatur durchgesetzt) wurde. Ferner: Unter Berufung auf Niels Bohr und Werner Heisenberg (letzterer übrigens bei Enzensberger unterrepräsentiert) kann man durchaus folgern, dass 
das Verlangen nach positivistischer "Genauigkeit“ und die oberlehrerhaft strenge Forderung nach Vermeidung alles „Spekulativen“ ihrerseits Unreflektiert-Einseitiges an den Tag gefördert hat. Wir stünden dann, mit unserem Interesse an der Neubegründung einer „Dritten Kultur“, doch tatsächlich vor einer neuen Romantik? Vor der Rückbesinnung auf Ursprünglich-Mythisches gar, im Sinne von Botho Strau $\beta$ ? Hans Magnus Enzensberger jedenfalls meint dies, und seinem geschulten Kopf fallen die Argumente nur so zu: „Auf die Gefahr hin, manchen ,harten "Verteidiger des Status quo vor den Kopf zu stossen, kann man die Behauptung riskieren, dass die avancierteste Wissenschaft zur zeitgenössischen Form des Mythos geworden ist. Gleichsam hinter dem Rücken der eigenen Ideologie kehren in ihren Konzeptionen, von den meisten Forschern unbemerkt, alle Ursprungsfragen, Träume und Albträume der Menschheit in neuer Gestalt wieder. Ihre Metaphern sind nur der sprachliche Ausdruck dieser Mythenproduktion. " ${ }^{\text {In }}$ der Tat würde sich dann ein Kreis Tat schliessen; gar eine Rückkehr der abendländischen ratio zu ursprünglich griechischer Tiefe sich neu ereignen? Der Traum des Novalis in seiner letzten Verwirklichung in der Novellen-Literatur des Botho Strauß? Die Uckermark ein neues Jena? Jedenfalls gilt: Die blosse Berufung auf eine positivistische „Wissenschaftlichkeit“ muss überwunden und die WeltErweiterung, die die neueste Physik bewirkt hat (und deren Konsequenzen sie womöglich nicht einmal selbst zu Ende reflektiert hat) muss in die transdisziplinäre Theoriebildung integriert werden. Wenn Raum und Zeit nicht mehr unabhängig voneinander zu denken sind, warum sollen Humaniora und Naturwissenschaften nicht ebenfalls notwendig interagieren? Die strikte Interdisziplinarität einerseits und die umfassende Erkenntnisleistung der Metapher andererseits stellen jene Organe zur Verfügung, die einer neuen Osmose der Disziplinen gerecht $\mathrm{zu}$ werden versprechen. Sie könnten eine Apotheose der „Dritten Kultur" evozieren, wie sie hier angestrebt wird, mit den Beispielfällen von Franz Kafka (und Michel Houellebecq) als ihren Haupt-Autoren.

Bereits Einstein selbst wusste darum. Der Physiker sah einerseits Kants und Humes Kritik an der metaphysischen Denkweise als berechtigt an. Doch im Eingedenken daran, wie kompliziert der Kosmos sich darstellte, musste Einstein auch bestimmten metaphysischen und ,poetischen“ Elementen in der Theorieentwicklung ihr Recht zugestehen. Für Kafka jedenfalls gilt: Als „Blutsbruder“ des Romantikers Kleist erstatten oft Fragmente, als solche gewollte, das vormals „abgerundete Ganze“. Auch das hat Adorno in seinen Aufzeichnungen gesehen.

\footnotetext{
${ }^{9}$ Hans M. Enzensberger, a. a. O., S. 89.
} 
Darin erwies sich der Romanverfasser Kafka als ein Epoche schaffender Autor, neue Standards setzend in Romanen, die immer auch Fragment geblieben sind und dennoch les- und deutbar ausfielen, wie immer sie sich in vexierender Polyvalenz präsentieren. Auch hierin muss Theodor Wiesengrund Adorno das letzte Wort zugestanden werden: „Es ist eine Parabolik, zu der der Schlüssel entwendet ward ... Jeder Satz spricht: deute mich, und keiner will es dulden." Und: Franz Kafka sei die „Schrift gewordene Turandot.“" ${ }^{10}$ Die chinesische Prinzessin wies bekanntlich alle Freier ab, in jüngerer Zeit sogar den eloquenten und schönen Jaques Derrida. ${ }^{11}$ Und hatte doch keinen Mangel an ihnen. Im Gegenteil, sie agierte schließlich im Gleichtakt mit Puccinis verführerischer Musik, dabei inspiriert von einem fernöstlich aufgefassten Mozart.

${ }^{10}$ Theodor W. Adorno, Aufzeichnungen zu Kafka, a. a. O., S. 304.

${ }^{11}$ Siehe dazu Laurent Binet, Die siebente Sprachfunktion, Hamburg 2017; und im Zusammenhang damit Bernd Neumann, Der andere Franz Kafka, Würzburg 2018, S. 20 ff. 
Open Access Dieses Kapitel wird unter der Creative Commons Namensnennung 4.0 International Lizenz (http://creativecommons.org/licenses/by/4.0/deed.de) veröffentlicht, welche die Nutzung, Vervielfältigung, Bearbeitung, Verbreitung und Wiedergabe in jeglichem Medium und Format erlaubt, sofern Sie den/die ursprünglichen Autor(en) und die Quelle ordnungsgemäß nennen, einen Link zur Creative Commons Lizenz beifügen und angeben, ob Änderungen vorgenommen wurden.

Die in diesem Kapitel enthaltenen Bilder und sonstiges Drittmaterial unterliegen ebenfalls der genannten Creative Commons Lizenz, sofern sich aus der Abbildungslegende nichts anderes ergibt. Sofern das betreffende Material nicht unter der genannten Creative Commons Lizenz steht und die betreffende Handlung nicht nach gesetzlichen Vorschriften erlaubt ist, ist für die oben aufgeführten Weiterverwendungen des Materials die Einwilligung des jeweiligen Rechteinhabers einzuholen.

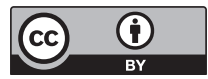

